\title{
Fractionation of Ovarian Follicles and in vitro Oocyte Maturation and Ovulation Assay of Ciona intestinalis Type A
}

Shin Matsubara ${ }^{1,2}$, Akira Shiraishi ${ }^{1}$, Tomohiro Osugi ${ }^{1}$, Tsuyoshi Kawada ${ }^{1}$ and Honoo Satake ${ }^{1, \star}$

\author{
${ }^{1}$ Bioorganic Research Institute, Suntory Foundation for Life Sciences, Kyoto 619-0284, Japan; \\ ${ }^{2}$ Research Fellow of Japan Society for the Promotion of Science, Tokyo, Japan \\ *For correspondence: satake@sunbor.or.jp
}

\begin{abstract}
[Abstract] Ascidians are the closest living relatives of vertebrates (Delsuc et al., 2006; Satoh et al., 2014) and are important for the evolutionary study of the ovarian follicle development including oocyte maturation and ovulation. However, neither the endogenous factors nor the molecular mechanisms underlying the oocyte maturation and ovulation had been elucidated mainly due to the lack of efficient procedure for isolating ovarian follicles. Here, we present the protocol for the effective fractionation and isolation of the ovarian follicle of Ciona intestinalis type A using stainless steel sieves with various particle size-meshes, and the simple incubation method of Ciona follicles for evaluating oocyte maturation and ovulation. Combined with the RNA-seq data from each fraction, the current methods lead us to investigate ovarian follicle development including oocyte maturation and ovulation in a stagespecific manner.
\end{abstract}

Keywords: Ciona intestinalis Type A, Ovary, Follicle isolation, Fractionation, Oocyte maturation, Germinal vesicle breakdown (GVBD), Ovulation

[Background] Ciona ovarian follicles are classified into four major developmental stages: stage I (previtellogenic), stage II (vitellogenic), stage III (post-vitellogenic, pre-GVBD), and stage IV (post-GVBD, ovulated) follicles (Figure 1; Prodon et al., 2006; Silvestre et al., 2009). The ovary of adult ascidians contains numerous follicles at entire developmental stages, and thus, isolation of individual follicles at specific stage is time-consuming and troublesome. This hindered functional analyses in follicular development and suggests requirement for efficient method of follicle isolation. Here we provide an effective fractionation method that a mixture of every developmental stage of follicles can be fractionated using a series of stainless steel sieves with various particle-size meshes (20-150 $\mu \mathrm{m})$. The current fractionation method enables us to isolate the entire developmental stages of follicles easily and quickly. Moreover, oocyte maturation and ovulation can be evaluated by germinal vesicle breakdown (i.e., GVBD) and rupture of follicular cell layer, respectively (Figure 1). Therefore, a simple incubation method of immature/pre-ovulatory follicle is useful for elucidating the endogenous regulator and the underlying molecular mechanisms oocyte maturation and ovulation as in Matsubara et al., 2019. Furthermore, transcriptomic data of the entire developmental stages of Ciona follicles, combined with fractionation of respective developmental-stage follicles, lead to the comprehensive understanding of not only oocyte maturation and ovulation but also the entire developmental mechanisms of Ciona follicles. The present fractionation and culture systems are also expected to be applicable to follicles of other aquatic animals 
including jellyfish, starfish, sea urchin and fish, and will contribute a great deal to advances in the study on oocyte maturation and ovulation in various organisms.

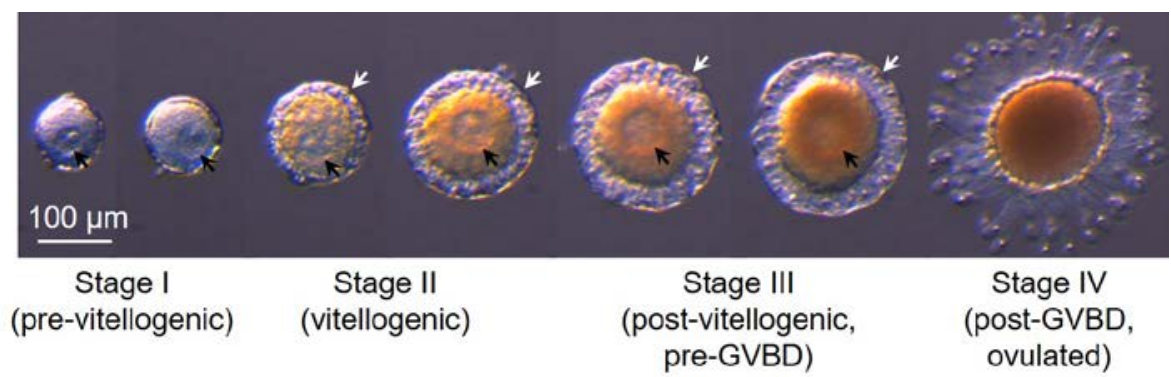

Figure 1. Developmental stages of Ciona ovarian follicles. Immature/pre-ovulatory (stage I-III) follicles show a germinal vesicle (GV, black arrow) and are enveloped by follicular cells and outer follicular cell (OFc) layer (white arrow). Oocytes mature via GV breakdown (GVBD) and ovulate via rupture of OFc layer. The ovary of adult ascidians contains numerous follicles of the entire developmental stages.

\section{Materials and Reagents}

1. 50-ml tube (IWAKI AGC TECHNO GLASS, catalog number: 2345-050)

2. 1.5-ml tube (Eppendorf, catalog number: 0030125215)

3. 60-mm dish (Corning, catalog number: 353002 for follicles before fractionation or Greiner BioOne, catalog number: 628979 for follicles after fractionation)

4. 35-mm dish (IWAKI AGC TECHNO GLASS, catalog number: 1000-035)

5. Pipette tips:

1,000- $\mu$ lips (Thermo Scientific, catalog number: H-111-R100NS-Q)

10- $\mu$ l tips (Greiner Bio-One, catalog number: 771251)

6. 1-L filter (Corning, catalog number: 431098)

7. 1-L storage bottle (Corning, catalog number: 430518)

8. 50-ml pipette (AS ONE, catalog number: 2-4132-06)

9. Adult ascidians (Ciona intestinalis Type A) cultivated in Maizuru Fisheries Research Station of Kyoto University or Misaki Marine Biological Station of the University of Tokyo

10. Positive factors of oocyte maturation and ovulation (e.g., Synthetic Ciona vasopressin (CiVP) peptide (Sequence: CFFRDCSNMDWYR, disulfide bridge: 1-6, Kawada et al., 2008)

11. Negative factors of oocyte maturation and ovulation, e.g.,

U0126 (Promega, catalog number: V1121)

Ro-3306 (Abcam, catalog number: ab141491)

MMP-2/MMP-9 inhibitor II (Calbiochem, catalog number: 444249)

12. DMSO (Nacalai tesque, catalog number: 13407-45)

13. REI-SEA marine powder (REI-SEA IWAKI, catalog number: 274513)

14. Artificial sea water (ASW) (see Recipes) 
15. $1 \mathrm{mM}$ CiVP (see Recipes)

16. $10 \mathrm{mM} \cup 0126$ (see Recipes)

17. $10 \mathrm{mM}$ Ro-3306 (see Recipes)

18. $10 \mathrm{mM}$ MMP-2/MMP-9 inhibitor II (see Recipes)

\section{Equipment}

1. 1-L beaker (IWAKI AGC TECHNO GLASS, catalog number: 0988-01-01-66)

2. Water tank (width $75 \mathrm{~cm}$, depth $45 \mathrm{~cm}$, height $45 \mathrm{~cm}$ )

3. Water pump (REI-SEA IWAKI, model: RMD-151, maximum flow rate: $19 \mathrm{~L} / \mathrm{min}$ )

4. Air pump (TECHNO TAKATSUKI, model: HP-30, maximum flow rate: $30 \mathrm{~L} / \mathrm{min}$ )

5. Portable pipet controller (Drummond, catalog number: 4-040-101-J)

6. Forceps (Rubis, catalog numbers: 2-5149-08, 2-5149-12)

7. Scissors (AS ONE, catalog number: 2-539-11)

8. Stainless steel sieves (TOKYO SCREEN Co., LTD., catalog numbers: TS-50-20-39 (Aperture: $180 \mu \mathrm{m}$ ); TS-50-20-41 (Aperture: $150 \mu \mathrm{m}$ ); TS-50-20-45 (Aperture: $90 \mu \mathrm{m}$ ); TS-50-20-47 (Aperture: $63 \mu \mathrm{m}$ ); TS-50-20-50 (Aperture: $38 \mu \mathrm{m}$ ); TS-50-20-53 (Aperture: $20 \mu \mathrm{m}$ ), https://www.tokyo-screen.com/.en/)

9. Stereo microscopes (ZEISS, model: SteREO Discovery V8 for dissecting of the ovary and isolating follicles; Leica Microsystems, model: Leica M205 FA equipped with Leica DFC 310 FX camera for recording follicles before and after incubation)

10. Microscale slide (WATSON Bio Lab, catalog number: 177-401C)

\section{Software}

1. Leica Aplication Suite Advanced Fluorescence (LAS-AF)

2. Fiji software (http://fiji.scl; Schindelin et al., 2012)

3. Microsoft Excel (Microsoft Corporation)

\section{Procedure}

A. Isolation of Ciona follicles from the ovary

1. Keep the adult ascidians (20-60 individuals) in a water tank with constant lighting, ASW circulating (19 L/min), and air bubbling (moderate level) at $18^{\circ} \mathrm{C}$ for four or five days.

2. Isolate the ovaries as in Figure 2 from 6-8 ascidians and collect them into a 50-ml tube with ASW.

Note: All the procedures should be performed at room temperature $\left(20^{\circ} \mathrm{C}\right)$. 

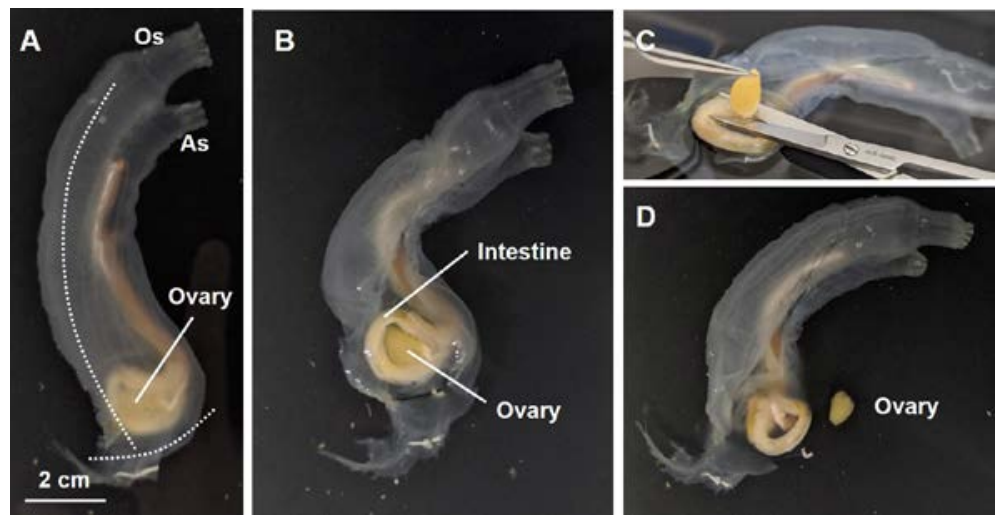

Figure 2. Enucleation of the ovary from an ascidian. A. Overview of an adult ascidian. Os, oral siphon; As, atrial siphon. B. Incise the transparent tunic [dashed line in $(A)$ ] with scissors and open the tunic with forceps. C. Pick up the ovary with forceps and isolate the ovary with scissors. D. Isolated ovary and the remaining tissues.

3. Wash the ovaries three times each with $45 \mathrm{ml}$ of ASW by inverting tube several times and gravity fall of the ovary.

4. Collect the ovaries into the normal 60-mm dish (Corning) with 5-6 ml of ASW (Figure 3A).

5. Incise the ovaries using two forceps under a stereo microscope (ZEISS, Video 1).

Note: If you do not need stage IV (matured and ovulated) follicles (Figures 3B-3C), remove them by gently shaking the ovaries using a forceps.

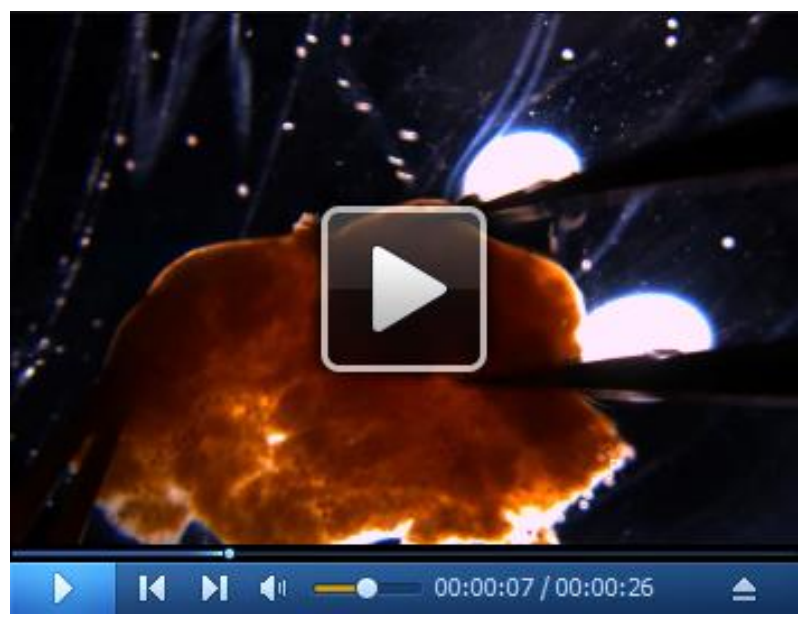

Video 1. Incising the Ciona ovary. The Ciona ovary is carefully incised and gently shaken using two forceps.

6. Transfer the ovaries to another $60-\mathrm{mm}$ dish with $5-6 \mathrm{ml}$ of new ASW.

7. Rip the ovaries at many sites using two forceps and release the immature/pre-ovulatory follicles by shaking the ovaries gently.

8. Repeat Steps A6-A7 five or six times (Figures 3D-3H) and collect all of the isolated follicles (Figure $3 \mathrm{l}$ ) into the first sieve (see below, Figure 4). 


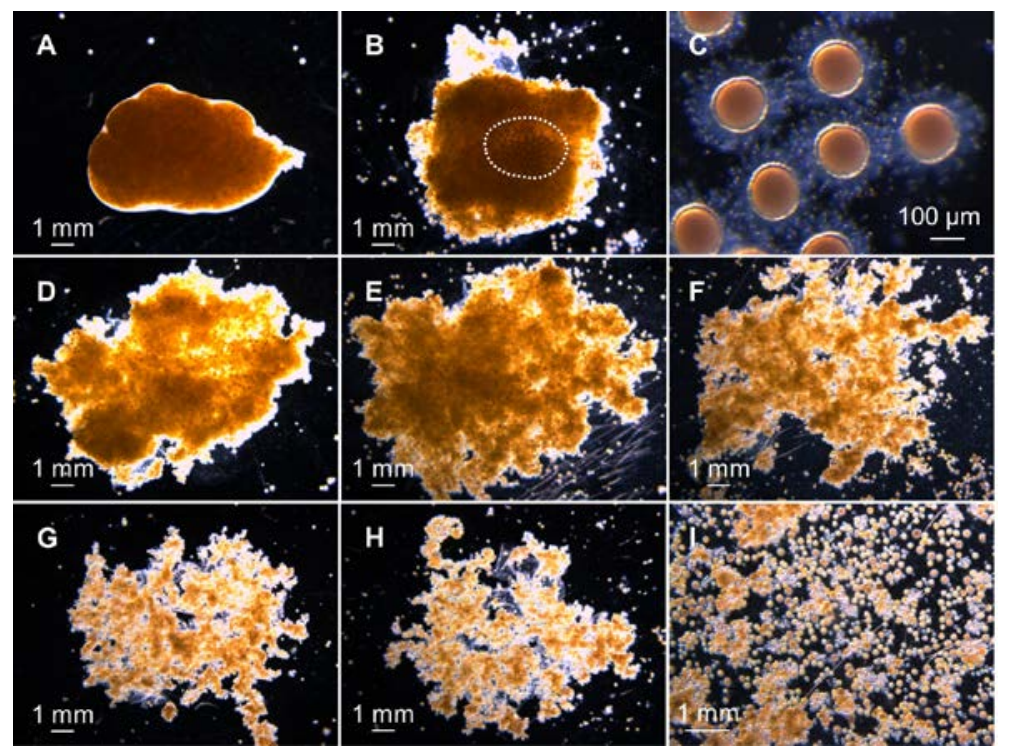

Figure 3. Dissection of the Ciona ovary and collection of follicles. A. An isolated ovary from an ascidian. B. The ovary was incised using two forceps. Dark dots represent stage IV follicles (dashed line). C. Enlarged image of stage IV follicles in (B). D-H. Series of dissecting the ovary. I. Resulting collected follicles at stage I-IV.
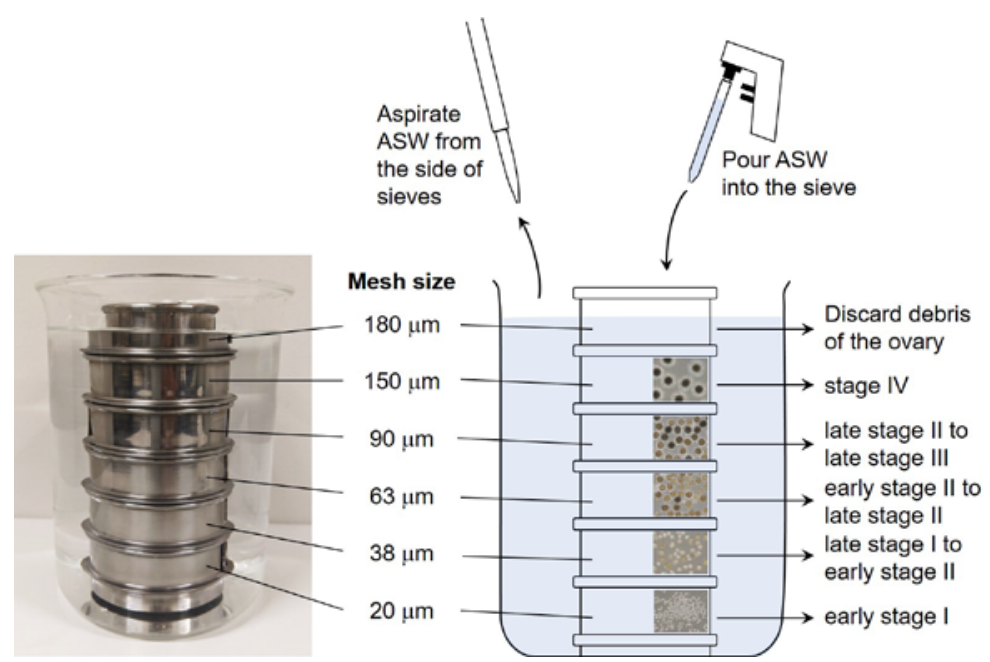

Figure 4. Series of stainless steel sieves in ASW. The series of sieves were stacked in ASW without any bubbles in order from the lowest one (20- $\mu \mathrm{m}$ mesh sieve). Fractionated follicles were washed by pouring and aspirating ASW, and collected at each sieve.

B. Fractionation and isolation of Ciona follicles

1. Prepare a series of stainless steel sieves in a 1-L beaker with ASW (Figure 4).

Note: Do not get air bubbles between the meshes.

2. Transfer all of the collected follicles (Figure 3l) into the upper sieve (Figure 4, $180 \mu \mathrm{m}$ ).

3. Wash the follicles by pouring $50-60 \mathrm{ml}$ of ASW into the stainless sieve gently, and aspirate ASW from the side of sieves (Figure 4).

Note: Keep the surface of ASW at about 1-cm height from the mesh not to dry the follicles. 
4. Repeat Step B3 four or five times and collect the follicles from the sieve to the 60-mm cellrepellent dish (Greiner Bio-One) by pipetting with $1,000 \mu$ of pipette tip.

Note: Do not use the normal 60-mm dish (Corning), given that pre-ovulatory follicles stick to it.

5. Aspirate ASW to the 1-cm height from the mesh of the lower sieve and remove the upper sieve. Note: The 180- $\mu$ m sieve mainly includes debris of the ovary.

6. Repeat Steps B3-B5 and collect developmental-stage follicles of interest.

Note: Representative images of entire developmental stages of fractionated follicles (including stages I-IV) are shown in Figure 2A in Matsubara et al. (2019).

7. Isolate the immature/pre-ovulatory follicles (approximately 20 follicles per sample) using a 10$\mu \mathrm{m}$ pipette tip (Greiner Bio-One) under the stereo microscope (ZEISS).

Note: Immature/pre-ovulatory follicles can be isolated from the $150-\mu \mathrm{m}$ or $90-\mu \mathrm{m}$ fraction of stage IV-removed samples, when follicles are used for maturation and ovulation (see Figure $2 B$ in Matsubara et al., 2019).

8. Transfer the 20 follicles to the center of a new $35-\mathrm{mm}$ dish with $2 \mathrm{ml}$ of ASW, and record the position of every follicle (Figure 5 , left).

Note: Do not use a 60-mm cell-repellent dish (Greiner Bio-One). Movement of follicles makes it difficult to distinguish individual follicles.

9. Take images of each follicle (Figure 5, right) and microscale slide using the stereo microscope (Leica) and LAS-AF software at the maximum magnification (160x).

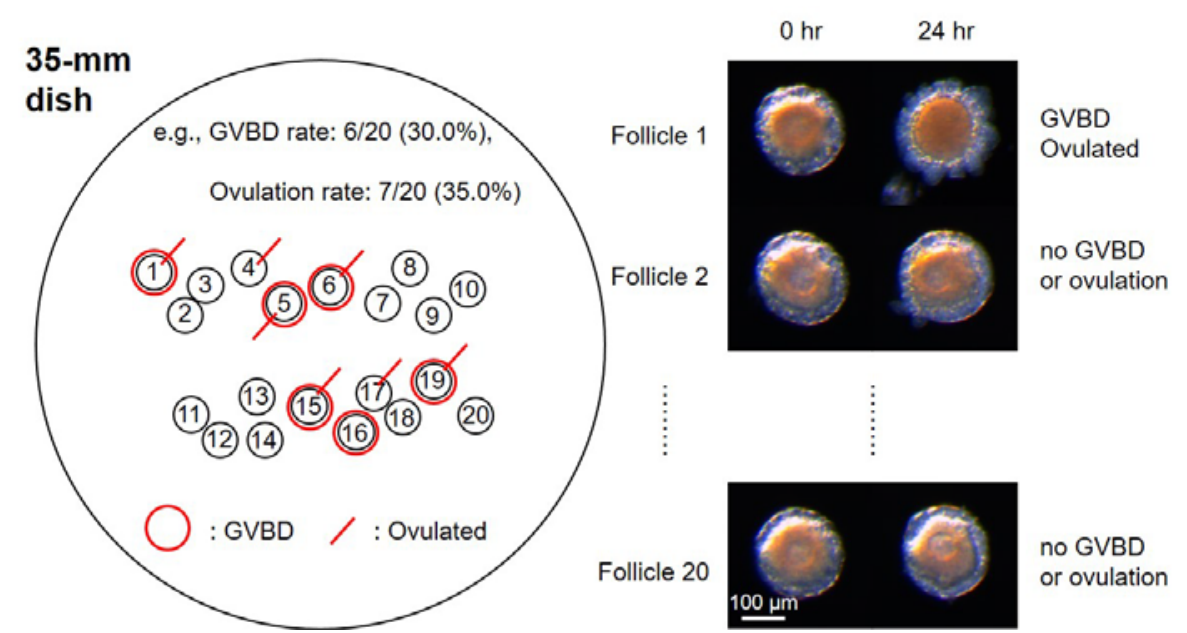

Figure 5. Recording follicle positions and states of GVBD and ovulation. Isolated follicles were attached to the 35-mm dish, and their positions are recorded (left). Images of individual follicle were taken before and after incubation (right). GVBD- and/or ovulated-follicles were marked and the ratios were calculated (left).

C. Induction of Ciona oocyte maturation and ovulation by synthetic CiVP

1. Isolate the approximately 40 of late stage II follicles (20 each for Control and CiVP).

2. Mix $15 \mu \mathrm{l}$ of the synthetic CiVP (1 mM) peptide or sterile MilliQ in $1 \mathrm{ml}$ of ASW in a 1.5-ml tube. 
3. Add the peptide or sterile MilliQ to the $35-\mathrm{mm}$ dish of follicles and mix well gently.

4. Incubate the follicles for $24 \mathrm{~h}$ at $20^{\circ} \mathrm{C}$.

5. Take images of follicles with a microscale slide using a stereo microscope (Leica) at the maximum magnification (x160) and record the position of every follicle (Figure 5).

6. Evaluate the GVBD and/or ovulation state of each follicle.

Note: GVBD and ovulation were evaluated by disappearance of germinal vesicle and rupturing of the outer follicular cell layer followed by release of the ovulated follicle, respectively.

D. Inhibition of Ciona oocyte maturation and ovulation by chemicals (e.g., MEK inhibitor, U0126)

1. Isolate the approximately 40 early stage III follicles (20 each for Control and U0126). Note: Isolate late stage III follicles for inhibition of oocyte maturation and ovulation using $10 \mathrm{mM}$ Ro-3306 and 10 mM MMP-2/MMP-9 inhibitor II, respectively.

2. Mix $3 \mu \mathrm{l}$ of $\mathrm{U} 0126(10 \mathrm{mM})$ or DMSO in $1 \mathrm{ml}$ of ASW in a 1.5-ml tube.

3. Add the chemical or DMSO to follicles in the $35-\mathrm{mm}$ dish and mix it well gently.

4. Incubate the follicles for $24 \mathrm{~h}$ at $20^{\circ} \mathrm{C}$.

5. Take images of follicles with a microscale slide using a stereo microscope (Leica) at the maximum magnification (x160) and record the position of every follicle (Figure 5).

6. Evaluate the GVBD and/or ovulation state of each follicle.

\section{Data analysis}

Export every image of follicles and a microscale slide as JPEG or TIFF files and open it with Fiji software. Set a $100-\mu \mathrm{m}$ scale of the microscale slide as a reference of distance using the line selection tool. Surround the follicle with the oval selection tool and measure the size of each follicle before incubation by the length of the major axis of oval fit (Figure 6A) and export the results to Microsoft Excel (Figure 6B). Analyze the follicle-size distribution between experimental groups (e.g., Control vs CiVP) by Student's $t$-test and confirm no significant difference (Figure 6B). Annotate GVBD and/or ovulation of each follicle to the size information, calculate GVBD and ovulation rates (Figure 6B), and indicate the results as graph (Figure 6C). Repeat the experiments three to six times and perform Student's $t$-test in Microsoft Excel (Matsubara et al., 2019). 
A

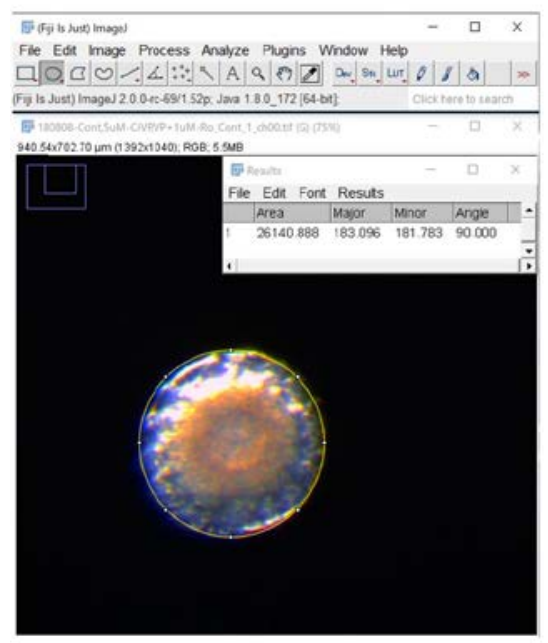

B

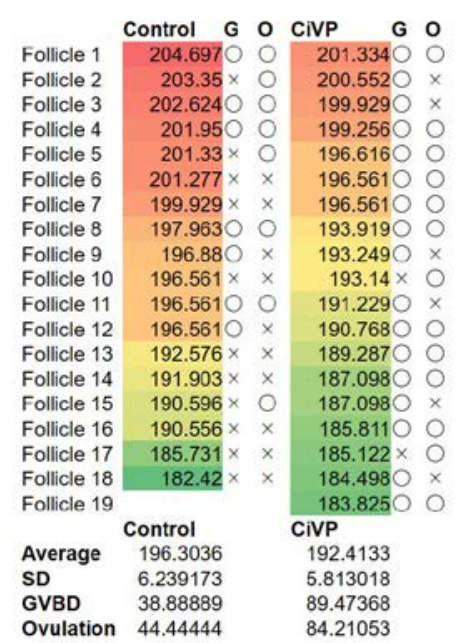

C

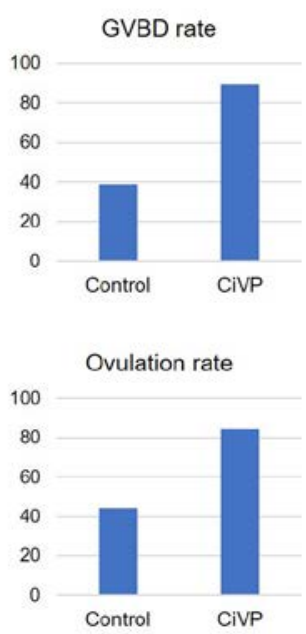

Figure 6. Data analysis of the in vitro oocyte maturation and ovulation assay. A. Size of each follicle was measured using Ellipse fit of Fiji software. B. Information of GVBD (G) and ovulation (O) of each follicle was annotated to the follicle number and size. C. GVBD and Ovulation rates were calculated and indicated by bar graphs.

\section{Recipes}

1. ASW (Artificial sea water)

Dissolve 200-g REI-SEA marine powder in 5-L tap water

Adjust specific gravity to 1.026 and filter sterlize

Store in a 1-L storage bottle at $20^{\circ} \mathrm{C}$ up to 1 month

2. $1 \mathrm{mM}$ CiVP

Dissolve in sterile MilliQ

Store at $-20^{\circ} \mathrm{C}$ up to 6 months

3. $10 \mathrm{mM} \cup 0126$

Dissolve in DMSO

Store at $-20^{\circ} \mathrm{C}$ in the dark up to 2 weeks

4. $10 \mathrm{mM}$ Ro-3306

Dissolve in DMSO

Store at $-20^{\circ} \mathrm{C}$ up to 6 months

5. $10 \mathrm{mM}$ MMP-2/MMP-9 inhibitor II

Dissolve in DMSO

Store at $-20^{\circ} \mathrm{C}$ for up to 3 months 


\section{Acknowledgments}

We gratefully acknowledge the National Bio-Resource Project (Maizuru Fisheries Research Station of Kyoto University or Misaki Marine Biological Station of the University of Tokyo) for providing ascidians. This work was supported in part by grants from the Japan Society for the Promotion of Science to SM (JP16K18581 and JP17J10624) and HS (JP16KK07430).

\section{Competing interests}

The authors declare that no competing interests exist.

\section{References}

1. Delsuc, F., Brinkmann, H., Chourrout, D. and Philippe, H. (2006). Tunicates and not cephalochordates are the closest living relatives of vertebrates. Nature 439(7079): 965-968.

2. Kawada, T., Sekiguchi, T., Itoh, Y., Ogasawara, M. and Satake, H. (2008). Characterization of a novel vasopressin/oxytocin superfamily peptide and its receptor from an ascidian, Ciona intestinalis. Peptides 29(10): 1672-1678.

3. Matsubara, S., Shiraishi, A., Osugi, T., Kawada, T. and Satake, H. (2019). The regulation of oocyte maturation and ovulation in the closest sister group of vertebrates. Elife 8: e49062.

4. Prodon, F., Chenevert, J. and Sardet, C. (2006). Establishment of animal-vegetal polarity during maturation in ascidian oocytes. Dev Biol 290(2): 297-311.

5. Satoh, N., Rokhsar, D. and Nishikawa, T. (2014). Chordate evolution and the three-phylum system. Proc Biol Sci 281(1794): 20141729.

6. Schindelin, J., Arganda-Carreras, I., Frise, E., Kaynig, V., Longair, M., Pietzsch, T., Preibisch, S., Rueden, C., Saalfeld, S., Schmid, B., Tinevez, J. Y., White, D. J., Hartenstein, V., Eliceiri, K., Tomancak, P. and Cardona, A. (2012). Fiji: an open-source platform for biological-image analysis. Nat Methods 9(7): 676-682.

7. Silvestre, F., Cuomo, A. and Tosti, E. (2009). Ion current activity and molecules modulating maturation and growth stages of ascidian (Ciona intestinalis) oocytes. Mol Reprod Dev 76(11): 1084-1093. 